\title{
FLUID QUEUES DRIVEN BY A BIRTH AND DEATH PROCESS WITH ALTERNATING FLOW RATES
}

\author{
P. R. PARTHASARATHY, K. V. VIJAYASHREE, AND R. B. LENIN
}

Received 18 January 2002 and in revised form 15 July 2002

Fluid queue driven by a birth and death process (BDP) with only one negative effective input rate has been considered in the literature. As an alternative, here we consider a fluid queue in which the input is characterized by a BDP with alternating positive and negative flow rates on a finite state space. Also, the BDP has two alternating arrival rates and two alternating service rates. Explicit expression for the distribution function of the buffer occupancy is obtained. The case where the state space is infinite is also discussed. Graphs are presented to visualize the buffer content distribution.

\section{Introduction}

Recent measurements have revealed that in high-speed telecommunication networks, like the ATM-based broadband ISDN, traffic conditions exhibit long-range dependence and burstiness over a wide range of time scales. Fluid models characterize such a traffic as a continuous stream with a parameterized flow rate. Whitt [6] establishes heavy-traffic stochastic process limits for fluid queue models with multiple on-off sources.

A fluid model that is typically used to model such a traffic is a Markov Modulated Fluid Model wherein the current state of the underlying Markov process determines the flow rate. Fluid models driven by finite state Markov processes that modulate the input rate in the fluid buffer have been analyzed by many authors. Lenin and Parthasarathy [2] provide closed-form expressions for the eigenvalues and eigenvectors for fluid queues driven by an $M / M / 1 / N$ queue. The case where the state space is infinite has been analyzed by van Doorn and Scheinhardt [5] for a birth and death process (BDP).

In most studies dealing with Markov modulated fluid queues, a single negative effective flow rate is assumed. Here, we consider a more general setting of a fluid queue driven by a BDP on a finite state space in which the flow rates are alternatively positive and negative. Our aim is to obtain the stationary distribution function of the buffer occupancy for this fluid model which is modulated by a BDP with two alternating arrival rates and two alternating service rates. This modulating Markov process can be visualized as a simple case of a two-state Markov Modulated Poisson Process which is characterized 
by a Markov process with an infinitesimal generator $\mathbf{Q}=\left[\begin{array}{cc}-\lambda_{1} & \lambda_{1} \\ \lambda_{2} & -\lambda_{2}\end{array}\right]$ and a diagonal matrix $\Lambda=\left[\begin{array}{cc}\lambda_{1} & 0 \\ 0 & \lambda_{2}\end{array}\right]$ of arrival probabilities, where $\lambda_{1}$ and $\lambda_{2}$ denote the rates of arrival when the traffic is bursty and slow, respectively. The case where the state space of the BDP is infinite is also discussed. Some interesting identities of tridiagonal determinants are used for the finite state space, and continued fraction methodology is employed for the infinite state space. Graphs are presented to visualize the buffer content distribution.

The model under consideration finds a wide range of application in modelling a communication switch as a fluid stochastic petri net in which two streams of traffic arrive (Horton et al. [1]). One stream is bursty with a high flow rate when the server is busy and the other stream is slow with a low flow rate when the workload of the server is less. We will designate the fluid commodity accumulating in the infinite capacity buffer as credit. It may be helpful to think of credit as the energy which the server gathers during lean traffic period and consumes when the traffic is bursty.

\section{Model description}

We consider an infinite capacity buffer which receives and releases fluid flows modulated by a BDP evolving in the background. We denote the background birth-death process by $\mathscr{X}:=\{X(t), t \geq 0\}$ taking values in the state space $\mathscr{Y}$, where $X(t)$ denotes the state of the process at time $t$. Let $\lambda_{n}$ and $\mu_{n}$ denote the mean arrival and service rates, respectively, when there are $n$ units in the system.

The flow rates of the fluid into and out of an infinite capacity buffer are determined by the actual state of the background process. Let $r_{j}$ denote the flow rate of the fluid when the background process is in state $j$. The rate of change of content of the buffer $C(t)$ when $X(t)=j$ is given by

$$
\frac{d C(t)}{d t}= \begin{cases}r_{j} & \text { if } C(t)>0 \\ 0 & \text { if } C(t)=0, r_{j}<0\end{cases}
$$

Clearly, the two-dimensional process $\{(X(t), C(t)), t \geq 0\}$ constitutes a Markov process which possesses a unique stationary distribution under a suitable stability condition.

The stationary state probabilities $p_{i}, i \in \mathscr{Y}$, of the BDP can be represented as

$$
p_{i}=\frac{\pi_{i}}{\sum_{j \in \mathscr{Y}} \pi_{j}}, \quad i \in \mathscr{Y}
$$

where $\pi_{i}=\lambda_{0} \lambda_{1} \cdots \lambda_{i-1} / \mu_{1} \mu_{2} \cdots \mu_{i}, i=1,2,3, \ldots$, and $\pi_{0}=1$ are called the potential coefficients. In order that a limit distribution for $C(t)$ exists as $t \rightarrow \infty$, the stationary net input rate should be negative, that is,

$$
\sum_{i=0}^{\infty} \pi_{i} r_{i}<0
$$


Letting

$$
F_{j}(t, u) \equiv P(X(t)=j, C(t) \leq u), \quad j \in \mathscr{Y}, t, u \geq 0
$$

the Kolmogorov forward equations for the Markov process $\{X(t), C(t)\}$ are given by

$$
\begin{aligned}
\frac{\partial F_{0}(t, u)}{\partial t}= & -r_{0} \frac{\partial F_{0}(t, u)}{\partial u}-\lambda_{0} F_{0}(t, u)+\mu_{1} F_{1}(t, u), \\
\frac{\partial F_{j}(t, u)}{\partial t}= & -r_{j} \frac{\partial F_{j}(t, u)}{\partial u}-\left(\lambda_{j}+\mu_{j}\right) F_{j}(t, u) \\
& +\lambda_{j-1} F_{j-1}(t, u)+\mu_{j+1} F_{j+1}(t, u), \quad j \in \mathscr{S} \backslash\{0\}, t, u \geq 0 .
\end{aligned}
$$

(See van Doorn and Scheinhardt [5]). When the process is in equilibrium, $\partial F_{j}(t, u) / \partial t \equiv$ 0 , and let $\lim _{t \rightarrow \infty} F_{j}(t, u) \equiv F_{j}(u)$.

\section{Finite state space}

This section deals with a fluid queue modulated by a finite BDP with state space $\mathscr{S}=$ $\{0,1,2, \ldots, N\}$. The system of equations governing the two-dimensional process $\{(X(t)$, $C(t)), t \geq 0\}$ in equilibrium is

$$
\begin{gathered}
r_{0} \frac{d F_{0}(u)}{d u}=-\lambda_{0} F_{0}(u)+\mu_{1} F_{1}(u), \\
r_{j} \frac{d F_{j}(u)}{d u}=\lambda_{j-1} F_{j-1}(u)-\left(\lambda_{j}+\mu_{j}\right) F_{j}(u)+\mu_{j+1} F_{j+1}(u), \quad \text { for } j \in \mathscr{S} \backslash\{0\}, u \geq 0 .
\end{gathered}
$$

In matrix notation (3.1) can be written as

$$
\frac{d \mathbf{F}(u)}{d u}=\mathbf{R}^{-1} \mathbf{Q}^{T} \mathbf{F}(u), \quad u \geq 0
$$

where $\mathbf{F}(u)=\left[F_{0}(u), F_{1}(u), \ldots, F_{N}(u)\right]^{T}, \mathbf{R}=\operatorname{diag}\left(r_{0}, r_{1}, \ldots, r_{N}\right)$, and

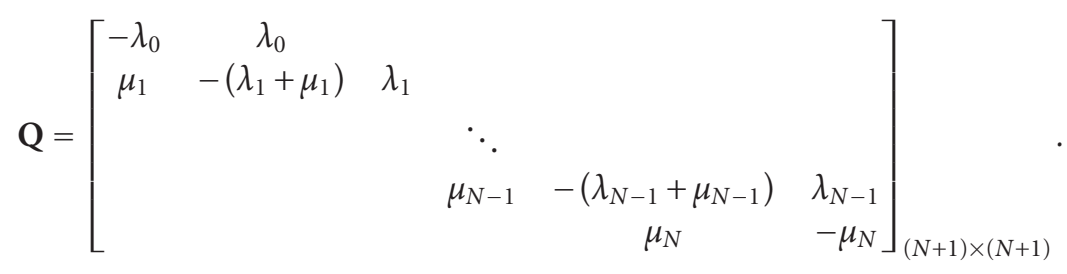


Hence

$$
\mathbf{R}^{-1} \mathbf{Q}^{T}=\left[\begin{array}{ccccc}
-\frac{\lambda_{0}}{r_{0}} & \frac{\mu_{1}}{r_{0}} & & & \\
\frac{\lambda_{0}}{r_{1}} & -\frac{\left(\lambda_{1}+\mu_{1}\right)}{r_{1}} & \frac{\mu_{2}}{r_{1}} & & \\
& & \ddots & & \\
& \frac{\lambda_{N-2}}{r_{N-1}} & -\frac{\left(\lambda_{N-1}+\mu_{N-1}\right)}{r_{N-1}} & \frac{\mu_{N}}{r_{N-1}} \\
& & \frac{\lambda_{N-1}}{r_{N}} & -\frac{\mu_{N}}{r_{N}}
\end{array}\right]_{(N+1) \times(N+1)}
$$

Mitra [3] has shown that $\mathbf{R}^{-1} \mathbf{Q}^{T}$ has exactly $N_{+}$negative eigenvalues, $N_{-}-1$ positive eigenvalues, and one zero-eigenvalue, where $N_{+}$is the cardinality of the set

$$
\mathscr{S}^{+}:=\left\{j \in \mathscr{S}: r_{j}>0\right\}
$$

and $N^{-}$is that of

$$
\mathscr{S}^{-}:=\left\{j \in \mathscr{S}: r_{j}<0\right\}
$$

Let $\xi_{j}, j=0,1,2, \ldots, N$, be the eigenvalues of the matrix $\mathbf{R}^{-1} \mathbf{Q}^{T}$ such that

$$
\xi_{j}<0, \quad j=0,1,2, \ldots, N_{+}-1, \quad \xi_{N^{+}}=0, \quad \xi_{j}>0, \quad j=N_{+}+1, \ldots, N .
$$

Since the content of the buffer increases when the net input rate of fluid flow into the buffer is positive, it follows that $F_{j}(u)$ must satisfy the boundary condition

$$
F_{j}(0)=0 \quad \text { for } j \in \mathscr{S}^{+} \text {. }
$$

Also, we have

$$
\lim _{u \rightarrow \infty} F_{j}(u)=p_{j} \quad \text { for } j \in \mathscr{Y},
$$

where the $p_{j}$ 's are the stationary state probabilities of the background BDP. The solution to the matrix equation (3.2) is given by

$$
F_{j}(u)=p_{j}+\sum_{l=0}^{N_{+}-1} \eta_{l, j} e^{\xi_{l} u}, \quad j \in \mathscr{S},
$$


where

$$
\eta_{l, j}=k_{l} \frac{B_{j}\left(\xi_{l}\right)}{c_{j 0}}, \quad c_{j 0}=\frac{\mu_{1} \mu_{2} \cdots \mu_{j}}{r_{0} r_{1} \cdots r_{j-1}} .
$$

The constants $k_{l}$ are obtained by solving

$$
p_{j}+\sum_{l=0}^{N+-1} k_{l} \frac{B_{j}\left(\xi_{l}\right)}{c_{j 0}}=0, \quad \text { for } j \in \mathscr{Y}^{+} .
$$

The polynomials $B_{j}(s)$ are defined recursively as

$$
\begin{gathered}
B_{0}(s)=1, \quad B_{1}(s)=s+\frac{\lambda_{0}}{r_{0}}, \\
B_{j}(s)=\left(s+\frac{\lambda_{j-1}+\mu_{j-1}}{r_{j-1}}\right) B_{j-1}(s)-\frac{\lambda_{j-2} \mu_{j-1}}{r_{j-2} r_{j-1}} B_{j-2}(s), \quad j=2,3,4, \ldots, N, \\
B_{N+1}(s)=\left(s+\frac{\mu_{N}}{r_{N}}\right) B_{N}(s)-\frac{\lambda_{N-1} \mu_{N}}{r_{N-1} r_{N}} B_{N-1}(s) .
\end{gathered}
$$

Also $B_{N+1}(s)=\operatorname{det}\left(s \mathbf{I}-\mathbf{R}^{-1} \mathbf{Q}^{T}\right)$ and $B_{j}(s)$ is the determinant obtained by considering the first $j$ rows and columns of $B_{N+1}(s)$. More specifically, we consider a fluid queue model with effective input rates $r_{2 j}<0$ and $r_{2 j+1}>0$ for $j=0,1,2, \ldots,(N-1) / 2$. Under this assumption, the system of equations involved in the determination of the constant $k_{l}$ is given in matrix form as

$$
\left[\begin{array}{cccc}
\frac{B_{1}\left(\xi_{0}\right)}{c_{10}} & \frac{B_{1}\left(\xi_{1}\right)}{c_{10}} & \cdots & \frac{B_{1}\left(\xi_{[N / 2]}\right)}{c_{10}} \\
\frac{B_{3}\left(\xi_{0}\right)}{c_{30}} & \frac{B_{3}\left(\xi_{1}\right)}{c_{30}} & \cdots & \frac{B_{3}\left(\xi_{[N / 2]}\right)}{c_{30}} \\
\frac{B_{5}\left(\xi_{0}\right)}{c_{50}} & \frac{B_{5}\left(\xi_{1}\right)}{c_{50}} & \cdots & \frac{B_{5}\left(\xi_{[N / 2]}\right)}{c_{50}} \\
\vdots & \vdots & & \vdots
\end{array}\right]_{[N / 2]}\left[\begin{array}{c}
k_{0} \\
k_{1} \\
k_{2} \\
\vdots
\end{array}\right]_{[N / 2]}=\left[\begin{array}{c}
p_{1} \\
p_{3} \\
p_{5} \\
\vdots
\end{array}\right]_{[N / 2]} .
$$

Hence the problem of determining the stationary distribution of the content in the fluid buffer is reduced to that of solving the above matrix equation via Cramer's rule.

We now give three examples to illustrate the above discussion. 
Example $3.1(N=1)$. The steady state probabilities for this two-state Markov process are given by $p_{0}=\mu_{1} /\left(\lambda_{0}+\mu_{1}\right)$ and $p_{1}=\lambda_{0} /\left(\lambda_{0}+\mu_{1}\right)$. It follows from (2.3) that the condition $\mu_{1} r_{0}+\lambda_{0} r_{1}<0$ ensures the stability of the Markov process $\{(X(t), C(t)), t \geq 0\}$.

The matrix $\mathbf{R}^{-1} \mathbf{Q}^{T}$ takes the form

$$
\mathbf{R}^{-1} \mathbf{Q}^{T}=\left[\begin{array}{cc}
-\frac{\lambda_{0}}{r_{0}} & \frac{\mu_{1}}{r_{0}} \\
\frac{\lambda_{0}}{r_{1}} & -\frac{\mu_{1}}{r_{1}}
\end{array}\right]
$$

with eigenvalues $\xi_{0}=-\left(\lambda_{0} / r_{0}+\mu_{1} / r_{1}\right)$ and $\xi_{1}=0$. The system has one negative root provided $\lambda_{0} / r_{0}+\mu_{1} / r_{1}>0$ (which follows from the stability condition). Further, $\eta_{00}=k_{0}$, $\eta_{01}=-k_{0} r_{0} / r_{1}$, and from $p_{1}+k_{0}\left(B_{1}\left(\xi_{0}\right) / c_{10}\right)=0$, we obtain $k_{0}=\left(r_{1} / r_{0}\right) p_{1}$. Therefore the final solution is given by

$$
\begin{aligned}
& F_{0}(u)=p_{0}+\frac{r_{1}}{r_{0}} p_{1} e^{-\left(\lambda_{0} / r_{0}+\mu_{1} / r_{1}\right) u}, \\
& F_{1}(u)=p_{1}-p_{1} e^{-\left(\lambda_{0} / r_{0}+\mu_{1} / r_{1}\right) u} .
\end{aligned}
$$

Observe that

$$
P(C(t)<u)=F_{0}(u)+F_{1}(u)=1-\left(1-\frac{r_{1}}{r_{0}}\right)\left(\frac{\lambda_{0}}{\lambda_{0}+\mu_{1}}\right) e^{-\left(\lambda_{0} / r_{0}+\mu_{1} / r_{1}\right) u}
$$

Example $3.2(N=2)$. The steady state probabilities of the modulating Markov process are given by

$$
\begin{array}{r}
p_{0}=\frac{\mu_{1} \mu_{2}}{\lambda_{0} \lambda_{1}+\lambda_{0} \mu_{2}+\mu_{1} \mu_{2}}, \\
p_{1}=\frac{\lambda_{0} \mu_{2}}{\lambda_{0} \lambda_{1}+\lambda_{0} \mu_{2}+\mu_{1} \mu_{2}}, \\
p_{2}=\frac{\lambda_{0} \lambda_{1}}{\lambda_{0} \lambda_{1}+\lambda_{0} \mu_{2}+\mu_{1} \mu_{2}} .
\end{array}
$$

It follows from (2.3) that the stability condition for the Markov process $\{(X(t), C(t)), t \geq$ $0\}$ is $\mu_{1} \mu_{2} r_{0}+\lambda_{0} \mu_{2} r_{1}+\lambda_{0} \lambda_{1} r_{2}<0$.

The matrix $\mathbf{R}^{-1} \mathbf{Q}^{T}$ takes the form

$$
\mathbf{R}^{-1} \mathbf{Q}^{T}=\left[\begin{array}{ccc}
-\frac{\lambda_{0}}{r_{0}} & \frac{\mu_{1}}{r_{0}} & \\
\frac{\lambda_{0}}{r_{1}} & -\frac{\lambda_{1}+\mu_{1}}{r_{1}} & \frac{\mu_{2}}{r_{1}} \\
& \frac{\lambda_{1}}{r_{2}} & -\frac{\mu_{2}}{r_{2}}
\end{array}\right]
$$


with eigenvalues given by

$$
\begin{aligned}
\xi_{0}= & -\frac{1}{2}\left(\frac{\lambda_{0}}{r_{0}}+\frac{\lambda_{1}}{r_{1}}+\frac{\mu_{1}}{r_{1}}+\frac{\mu_{2}}{r_{2}}\right) \\
& -\frac{1}{2} \sqrt{\left(\frac{\lambda_{0}}{r_{0}}+\frac{\lambda_{1}}{r_{1}}+\frac{\mu_{1}}{r_{1}}+\frac{\mu_{2}}{r_{2}}\right)^{2}-4\left(\frac{\lambda_{0} \lambda_{1}}{r_{0} r_{1}}+\frac{\mu_{1} \mu_{2}}{r_{1} r_{2}}+\frac{\lambda_{0} \mu_{2}}{r_{0} r_{2}}\right),} \\
\xi_{1}= & 0, \\
\xi_{2}= & -\frac{1}{2}\left(\frac{\lambda_{0}}{r_{0}}+\frac{\lambda_{1}}{r_{1}}+\frac{\mu_{1}}{r_{1}}+\frac{\mu_{2}}{r_{2}}\right) \\
& +\frac{1}{2} \sqrt{\left(\frac{\lambda_{0}}{r_{0}}+\frac{\lambda_{1}}{r_{1}}+\frac{\mu_{1}}{r_{1}}+\frac{\mu_{2}}{r_{2}}\right)^{2}-4\left(\frac{\lambda_{0} \lambda_{1}}{r_{0} r_{1}}+\frac{\mu_{1} \mu_{2}}{r_{1} r_{2}}+\frac{\lambda_{0} \mu_{2}}{r_{0} r_{2}}\right) .}
\end{aligned}
$$

The constant $k_{0}$ is determined as $k_{0}=-p_{1} \mu_{1} / r_{0} B_{1}\left(\xi_{0}\right)$, where

$$
\begin{gathered}
B_{0}(s)=1, \\
B_{1}(s)=\left(s+\frac{\lambda_{0}}{r_{0}}\right), \\
B_{2}(s)=\left(s+\frac{\lambda_{0}}{r_{0}}\right)\left(s+\frac{\lambda_{1}+\mu_{1}}{r_{1}}\right)-\frac{\lambda_{0} \mu_{1}}{r_{0} r_{1}} .
\end{gathered}
$$

Therefore, the stationary distribution of the buffer content is given by

$$
\begin{aligned}
& F_{0}(u)=p_{0}-p_{1} \frac{\mu_{1}}{r_{0}} \frac{1}{B_{1}\left(\xi_{0}\right)} e^{\xi_{0} u}, \\
& F_{1}(u)=p_{1}-p_{1} e^{\xi_{0} u}, \\
& F_{2}(u)=p_{2}-p_{1} \frac{r_{1}}{\mu_{2}} \frac{B_{2}\left(\xi_{0}\right)}{B_{1}\left(\xi_{0}\right)} e^{\xi_{0} u} .
\end{aligned}
$$

Observe that

$$
\begin{aligned}
P(C(t)<u) & =F_{0}(u)+F_{1}(u)+F_{2}(u) \\
& =1-p_{1}\left(1+\frac{\mu_{1}}{r_{0} B_{1}\left(\xi_{0}\right)}+\frac{r_{1}}{\mu_{2}} \frac{B_{2}\left(\xi_{0}\right)}{B_{1}\left(\xi_{0}\right)}\right) e^{\xi_{0} u} .
\end{aligned}
$$


In the above discussion, we considered two examples in the general case. The forthcoming example deals with the model in which the birth and death rates alternate between two constant values, with even number of states. The case with odd number of states does not lead to explicit expression for eigenvalues.

Example 3.3 (alternating rates). Consider a fluid queue driven by a single server queuing model with state space $\mathscr{Y}=\{0,1,2, \ldots, 2 n-1\}$ whose birth and death rates are given by

$$
\begin{aligned}
\lambda_{2 i} & =\lambda_{1}, \quad \mu_{2 i}=\mu_{2} \quad \text { for } i=1,2, \ldots, n-1, \\
\lambda_{2 i+1} & =\lambda_{2}, \quad \mu_{2 i+1}=\mu_{1} \quad \text { for } i=0,1, \ldots, n-2,
\end{aligned}
$$

with $\lambda_{0}=\lambda_{1}, \mu_{2 n-1}=\mu_{1}$, and the effective input rates are $r_{2 i}=r_{1}<0$ and $r_{2 i+1}=r_{2}>0$ for $i=0,1,2, \ldots, n-1$. If $\rho=\lambda_{1} \lambda_{2} / \mu_{1} \mu_{2}<1$, the steady state probabilities are given by

$$
\begin{gathered}
p_{2 k}=\left(\frac{\lambda_{1} \lambda_{2}}{\mu_{1} \mu_{2}}\right)^{k} p_{0}, \quad k=1,2,3, \ldots, n-1, \\
p_{2 k+1}=\frac{\lambda_{1}}{\mu_{1}}\left(\frac{\lambda_{1} \lambda_{2}}{\mu_{1} \mu_{2}}\right)^{k} p_{0}, \quad k=0,1,2, \ldots, n-1,
\end{gathered}
$$

with $p_{0}=\left(\mu_{1} /\left(\lambda_{1}+\mu_{1}\right)\right)\left((1-\rho) /\left(1-\rho^{n}\right)\right)$. From (2.3), it is observed that the condition $r_{1} \mu_{1}+r_{2} \lambda_{1}<0$ ensures the stability of the Markov process $\{(X(t), C(t)), t \geq 0\}$.

For this specific model, the matrix $\mathbf{R}^{-1} \mathbf{Q}^{T}$ takes the form

$$
\begin{aligned}
& \mathbf{R}^{-1} \mathbf{Q}^{T}=\left|\begin{array}{ccccc}
-\frac{\lambda_{1}}{r_{1}} & \frac{\mu_{1}}{r_{1}} & & \\
\frac{\lambda_{1}}{r_{2}} & -\frac{\lambda_{2}+\mu_{1}}{r_{2}} & \frac{\mu_{2}}{r_{2}} & & \\
& & \ddots & & \\
& \frac{\lambda_{2}}{r_{1}} & -\frac{\lambda_{1}+\mu_{2}}{r_{1}} & \frac{\mu_{1}}{r_{1}} \\
& & \frac{\lambda_{1}}{r_{2}} & -\frac{\mu_{1}}{r_{2}}
\end{array}\right|_{2 n} \\
& =\left|\begin{array}{ccccc}
-\frac{\lambda_{1}}{r_{1}} & \frac{\mu_{1}}{r_{2}} & & & \\
\frac{\lambda_{1}}{r_{1}} & -\frac{\lambda_{2}+\mu_{1}}{r_{2}} & \frac{\mu_{2}}{r_{1}} & & \\
& & \ddots & \\
& & \frac{\lambda_{2}}{r_{2}} & -\frac{\lambda_{1}+\mu_{2}}{r_{1}} & \frac{\mu_{1}}{r_{2}} \\
& & & \frac{\lambda_{1}}{r_{1}} & -\frac{\mu_{1}}{r_{2}}
\end{array}\right|_{2 n}
\end{aligned}
$$


Therefore,

$$
\begin{aligned}
& \left|s \mathbf{I}-\mathbf{R}^{-1} \mathbf{Q}^{T}\right|
\end{aligned}
$$

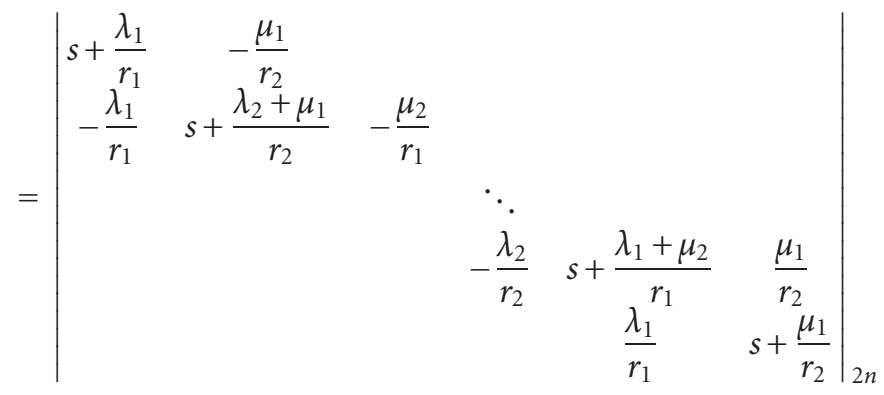

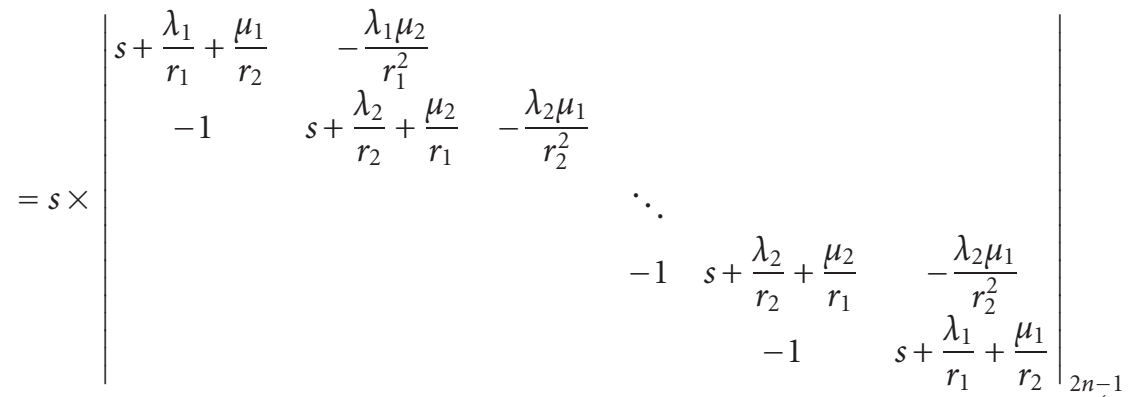

Using the Identities A.2, A.1, and A.3 given in the appendix in succession, with $\theta=s+$ $\lambda_{1} / r_{1}+\mu_{1} / r_{2}, \phi=s+\lambda_{2} / r_{2}+\mu_{2} / r_{1}$, and $\omega=\theta \phi-\lambda_{2} \mu_{1} / r_{2}^{2}-\lambda_{1} \mu_{2} / r_{1}^{2}$, we obtain

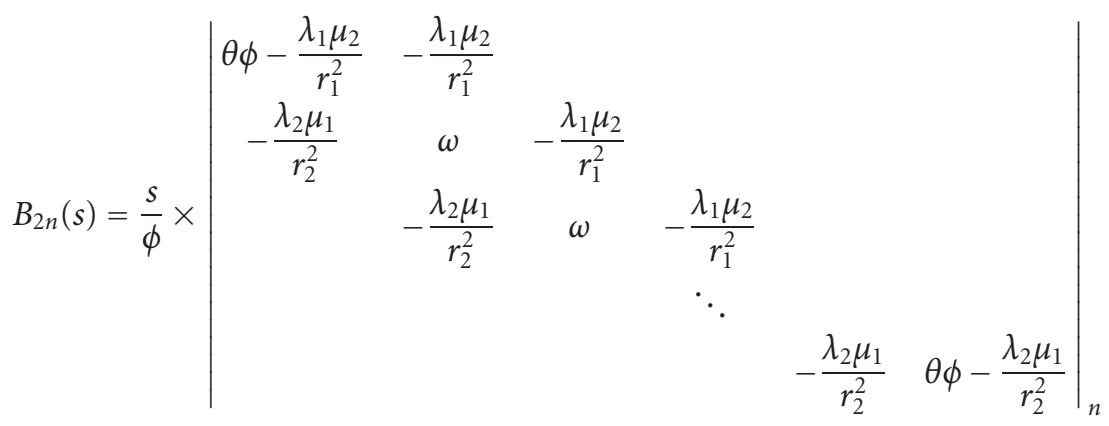

$$
\begin{aligned}
& =\frac{s}{\phi} \times \theta \phi \times\left|\begin{array}{ccccc}
\omega & -\frac{\lambda_{2} \mu_{1}}{r_{2}^{2}} & & & \\
-\frac{\lambda_{1} \mu_{2}}{r_{1}^{2}} & \omega & -\frac{\lambda_{2} \mu_{1}}{r_{2}^{2}} & & \\
& & & \ddots & \\
& & & -\frac{\lambda_{1} \mu_{2}}{r_{1}^{2}} & \omega
\end{array}\right|_{n-1}
\end{aligned}
$$


478 Fluid queues

$$
=s \theta \times \prod_{r=1}^{n-1}\left[\theta \phi-\frac{\lambda_{1} \mu_{2}}{r_{1}^{2}}-\frac{\lambda_{2} \mu_{1}}{r_{2}^{2}}-2 \sqrt{\frac{\lambda_{1} \lambda_{2} \mu_{1} \mu_{2}}{r_{1}^{2} r_{2}^{2}}} \cos \frac{r \pi}{n}\right] .
$$

Substituting for $\theta$ and $\phi$, we obtain

$$
\begin{aligned}
B_{2 n}(s)=s\left(s+\frac{\lambda_{1}}{r_{1}}+\frac{\mu_{1}}{r_{2}}\right) & \\
\times \prod_{r=1}^{n-1}[ & \left(s+\frac{\lambda_{1}}{r_{1}}+\frac{\mu_{1}}{r_{2}}\right)\left(s+\frac{\lambda_{2}}{r_{2}}+\frac{\mu_{2}}{r_{1}}\right)-\frac{\lambda_{1} \mu_{2}}{r_{1}^{2}} \\
& \left.-\frac{\lambda_{2} \mu_{1}}{r_{2}^{2}}-2 \frac{\sqrt{\lambda_{1} \lambda_{2} \mu_{1} \mu_{2}}}{r_{1} r_{2}} \cos \frac{r \pi}{n}\right] .
\end{aligned}
$$

We observe that $B_{2 n}(s)$ is zero when $-s$ is the eigenvalue of the tridiagonal matrix $\mathbf{R}^{-1} \mathbf{Q}^{T}$. Therefore, the eigenvalues of $\mathbf{R}^{-1} \mathbf{Q}^{T}$ are given by

$$
\begin{aligned}
& \xi_{0}=-\left(\frac{\lambda_{1}}{r_{1}}+\frac{\mu_{1}}{r_{2}}\right), \\
& \xi_{j}=\frac{1}{2}\left[-\left(\frac{\lambda_{1}+\mu_{2}}{r_{1}}+\frac{\lambda_{2}+\mu_{1}}{r_{2}}\right)-\left(\left(\frac{\lambda_{1}+\mu_{2}}{r_{1}}+\frac{\lambda_{2}+\mu_{1}}{r_{2}}\right)^{2}-4 \frac{\lambda_{1} \lambda_{2}}{r_{1} r_{2}}-4 \frac{\mu_{1} \mu_{2}}{r_{1} r_{2}}\right.\right. \\
& \left.\left.+8 \frac{\sqrt{\lambda_{1} \lambda_{2} \mu_{1} \mu_{2}}}{r_{1} r_{2}} \cos \frac{j \pi}{n}\right)^{1 / 2}\right], \quad j=1,2, \ldots, n-1, \\
& \xi_{n}=0, \\
& \xi_{j}=\frac{1}{2}\left[-\left(\frac{\lambda_{1}+\mu_{2}}{r_{1}}+\frac{\lambda_{2}+\mu_{1}}{r_{2}}\right)+\left(\left(\frac{\lambda_{1}+\mu_{2}}{r_{1}}+\frac{\lambda_{2}+\mu_{1}}{r_{2}}\right)^{2}-4 \frac{\lambda_{1} \lambda_{2}}{r_{1} r_{2}}-4 \frac{\mu_{1} \mu_{2}}{r_{1} r_{2}}\right.\right. \\
& \left.\left.+8 \frac{\sqrt{\lambda_{1} \lambda_{2} \mu_{1} \mu_{2}}}{r_{1} r_{2}} \cos \frac{j \pi}{n}\right)^{1 / 2}\right], \quad j=n+1, n+2, \ldots, 2 n-1 .
\end{aligned}
$$


P. R. Parthasarathy et al. 479

We give below closed-form expressions for the terms $B_{2 k}(s)$ and $B_{2 k+1}(s)$ for $k=0, \ldots, n-$ 1 , using the well-known identities of continuants given in the appendix. Consider

$$
\begin{aligned}
& B_{2 k}(s)=\left|\begin{array}{cccc}
s+\frac{\lambda_{1}}{r_{1}} & \frac{\mu_{1}}{r_{2}} & & \\
\frac{\lambda_{1}}{r_{1}} & s+\frac{\lambda_{2}+\mu_{1}}{r_{2}} & \frac{\mu_{2}}{r_{1}} & \\
& \ddots & & \\
& \frac{\lambda_{2}}{r_{2}} & s+\frac{\lambda_{1}+\mu_{2}}{r_{1}} & \frac{\mu_{1}}{r_{2}} \\
& & \frac{\lambda_{1}}{r_{1}} & s+\frac{\lambda_{2}+\mu_{1}}{r_{2}}
\end{array}\right|_{2 k} \\
& =\left|\begin{array}{cccc}
s+\frac{\lambda_{1}+\mu_{2}}{r_{1}} & \frac{\mu_{1}}{r_{2}} & & \\
\frac{\lambda_{1}}{r_{1}} & s+\frac{\lambda_{2}+\mu_{1}}{r_{2}} & \frac{\mu_{2}}{r_{1}} & \\
& \ddots & & \\
& \frac{\lambda_{2}}{r_{2}} & s+\frac{\lambda_{1}+\mu_{2}}{r_{1}} & \frac{\mu_{1}}{r_{2}} \\
& & \frac{\lambda_{1}}{r_{1}} & s+\frac{\lambda_{2}+\mu_{1}}{r_{2}}
\end{array}\right|_{2 k} \\
& -\frac{\mu_{2}}{r_{1}}\left|\begin{array}{cccc}
s+\frac{\lambda_{2}+\mu_{1}}{r_{2}} & \frac{\mu_{2}}{r_{1}} & & \\
\frac{\lambda_{2}}{r_{2}} & s+\frac{\lambda_{1}+\mu_{2}}{r_{1}} & \frac{\mu_{1}}{r_{2}} & \\
& \ddots & \\
& \frac{\lambda_{2}}{r_{2}} & s+\frac{\lambda_{1}+\mu_{2}}{r_{1}} & \frac{\mu_{1}}{r_{2}} \\
& & \frac{\lambda_{1}}{r_{1}} & s+\frac{\lambda_{2}+\mu_{1}}{r_{2}}
\end{array}\right|_{2 k-1}
\end{aligned}
$$


Using Identities A.2 and A.4,

$$
\begin{aligned}
& B_{2 k}(s)=\left|\begin{array}{rrrr}
\omega_{1}+\frac{\lambda_{2} \mu_{2}}{r_{1} r_{2}} & -\frac{\lambda_{1} \mu_{1}}{r_{1} r_{2}} & & \\
-\frac{\lambda_{2} \mu_{2}}{r_{1} r_{2}} & \omega_{1} & -\frac{\lambda_{1} \mu_{1}}{r_{1} r_{2}} & \\
& \ddots & & \\
& & \omega_{1} & -\frac{\lambda_{1} \mu_{1}}{r_{1} r_{2}} \\
& & -\frac{\lambda_{2} \mu_{2}}{r_{1} r_{2}} & \omega_{1}
\end{array}\right|_{k} \\
& -\frac{\mu_{2}}{r_{1} s+\lambda_{1}+\mu_{2}}\left|\begin{array}{rrrr}
\omega_{1}+\frac{\lambda_{2} \mu_{2}}{r_{1} r_{2}} & -\frac{\lambda_{1} \mu_{1}}{r_{1} r_{2}} & & \\
-\frac{\lambda_{2} \mu_{2}}{r_{1} r_{2}} & \omega_{1} & -\frac{\lambda_{1} \mu_{1}}{r_{1} r_{2}} & \\
& \ddots & & \\
& & \omega_{1} & -\frac{\lambda_{1} \mu_{1}}{r_{1} r_{2}} \\
& -\frac{\lambda_{2} \mu_{2}}{r_{1} r_{2}} & \omega_{1}+\frac{\lambda_{2} \mu_{2}}{r_{1} r_{2}}
\end{array}\right|_{k}
\end{aligned}
$$

where $\omega_{1}=\left(s+\left(\lambda_{1}+\mu_{2}\right) / r_{1}\right)\left(s+\left(\lambda_{2}+\mu_{1}\right) / r_{2}\right)-\lambda_{1} \mu_{1} / r_{1} r_{2}-\lambda_{2} \mu_{2} / r_{1} r_{2}$. Now, expanding the first determinant and using Identity A.1 for the second, we obtain

$$
B_{2 k}(s)=\left|\begin{array}{ccccc}
\omega_{1} & \frac{\lambda_{1} \mu_{1}}{r_{1} r_{2}} & & & \\
\frac{\lambda_{2} \mu_{2}}{r_{1} r_{2}} & \omega_{1} & \frac{\lambda_{1} \mu_{1}}{r_{1} r_{2}} & & \\
& & \ddots & & \\
& & & \omega_{1} & \frac{\lambda_{1} \mu_{1}}{r_{1} r_{2}} \\
& & \frac{\lambda_{2} \mu_{2}}{r_{1} r_{2}} & \omega_{1}
\end{array}\right|_{k}
$$




$$
\begin{aligned}
& +\frac{\lambda_{2} \mu_{2}}{r_{1} r_{2}}\left|\begin{array}{ccccc}
\omega_{1} & \frac{\lambda_{1} \mu_{1}}{r_{1} r_{2}} & & & \\
{\frac{\lambda}{2} \mu_{2}}_{1} & \omega_{1} & \frac{\lambda_{1} \mu_{1}}{r_{1} r_{2}} & & \\
& & & \ddots & \\
& & & \omega_{1} & \frac{\lambda_{1} \mu_{1}}{r_{1} r_{2}} \\
& & \frac{\lambda_{2} \mu_{2}}{r_{1} r_{2}} & \omega_{1}
\end{array}\right|_{k-1} \\
& -\frac{\mu_{2}}{r_{1}}\left(s+\frac{\lambda_{2}+\mu_{1}}{r_{2}}\right)\left|\begin{array}{ccccc}
\omega_{1} & \frac{\lambda_{2} \mu_{2}}{r_{1} r_{2}} & & & \\
\frac{\lambda_{1} \mu_{1}}{r_{1} r_{2}} & \omega_{1} & \frac{\lambda_{2} \mu_{2}}{r_{1} r_{2}} & & \\
& & \ddots & & \\
& & & \omega_{1} & \frac{\lambda_{2} \mu_{2}}{r_{1} r_{2}} \\
& & \frac{\lambda_{1} \mu_{1}}{r_{1} r_{2}} & \omega_{1}
\end{array}\right|_{k-1}
\end{aligned}
$$

Therefore, from Identity A.3, the quantity $B_{2 k}(s)$ can be expressed in closed form as follows:

$$
\begin{aligned}
B_{2 k}(s)= & \left(\frac{\lambda_{1} \lambda_{2} \mu_{1} \mu_{2}}{r_{1}^{2} r_{2}^{2}}\right)^{k / 2}\left[U_{k}\left(\frac{x}{2}\right)+\left(\frac{\lambda_{2} \mu_{2}}{\lambda_{1} \mu_{1}}\right)^{1 / 2} U_{k-1}\left(\frac{x}{2}\right)\right] \\
& -\frac{\mu_{2}}{r_{1}}\left(s+\frac{\lambda_{2}+\mu_{1}}{r_{2}}\right)\left(\frac{\lambda_{1} \lambda_{2} \mu_{1} \mu_{2}}{r_{1}^{2} r_{2}^{2}}\right)^{(k-1) / 2} U_{k-1}\left(\frac{x}{2}\right),
\end{aligned}
$$

where

$$
x=2\left[\frac{\left(r_{1} s+\lambda_{1}+\mu_{2}\right)\left(r_{2} s+\lambda_{2}+\mu_{1}\right)-\lambda_{1} \mu_{1}-\lambda_{2} \mu_{2}}{\sqrt{\lambda_{1} \lambda_{2} \mu_{1} \mu_{2}}}\right]
$$

and $U_{k}(x)$ is the Chebyshev polynomial of the second kind. Similarly, $B_{2 k+1}(s)$ can also be expressed as

$$
\begin{aligned}
B_{2 k+1}(s)= & \left(s+\frac{\lambda_{1}+\mu_{2}}{r_{1}}\right)\left(\frac{\lambda_{1} \lambda_{2} \mu_{1} \mu_{2}}{r_{1}^{2} r_{2}^{2}}\right)^{k / 2} U_{k}\left(\frac{x}{2}\right) \\
& -\frac{\mu_{2}}{r_{1}}\left(\frac{\lambda_{1} \lambda_{2} \mu_{1} \mu_{2}}{r_{1}^{2} r_{2}^{2}}\right)^{k / 2}\left[U_{k}\left(\frac{x}{2}\right)+\left(\frac{\lambda_{1} \mu_{1}}{\lambda_{2} \mu_{2}}\right)^{1 / 2} U_{k-1}\left(\frac{x}{2}\right)\right] .
\end{aligned}
$$


Further,

$$
c_{j 0}= \begin{cases}\left(\frac{\mu_{1} \mu_{2}}{r_{1} r_{2}}\right)^{k} & \text { if } j=2 k, \\ \frac{\mu_{1}}{r_{1}}\left(\frac{\mu_{1} \mu_{2}}{r_{1} r_{2}}\right)^{k} & \text { if } j=2 k+1 .\end{cases}
$$

Having determined $B_{j}(s)$ and the roots of $\mathbf{R}^{-1} \mathbf{Q}^{T}$ explicitly, the constants $k_{l}$, and hence the buffer occupancy distributions, are obtained using (3.10) and (3.14).

Remark 3.4. From the above discussion, we observe that, without loss of generality, $r_{1}$ and $r_{2}$ can be taken as -1 and +1 , respectively. This is because the solution remains unaltered when $\lambda_{1}\left(\mu_{2}\right)$ and $\lambda_{2}\left(\mu_{1}\right)$ are replaced by $-\lambda_{1} r_{1}\left(-\mu_{2} r_{1}\right)$ and $\lambda_{2} r_{2}\left(\mu_{1} r_{2}\right)$, respectively.

\section{Infinite state space}

In the previous section, we discussed the fluid queue model on a finite state space with alternating positive and negative flow rates. Similar analysis on an infinite state space does not lead to explicit expression for the buffer content distribution. Hence we analyze the fluid queue driven by an infinite state BDP with a single negative effective flow rate, say $r_{0}(<0)$. We employ the continued fraction methodology to obtain the stationary distribution of the content of the buffer. Let $F_{j}(t, u)$ represent the probability that there are $j$ units in the system and the content of the buffer does not exceed $u$ at time $t$, and let $\hat{F}_{j}(t, s)$ denote the corresponding Laplace transform. If $\lim _{t \rightarrow \infty} F_{j}(t, u)=F_{j}(u)$, then the governing system of differential-difference equations is

$$
\begin{gathered}
r_{0} F_{0}^{\prime}(u)=-\lambda_{0} F_{0}(u)+\mu_{1} F_{1}(u), \\
r_{j} F_{j}^{\prime}(u)=\lambda_{j-1} F_{j-1}(u)-\left(\lambda_{j}+\mu_{j}\right) F_{j}(u)+\mu_{j+1} F_{j+1}(u), \quad j=1,2, \ldots
\end{gathered}
$$

Using the initial condition $F_{0}(0)=a$, the Laplace transform of (4.1) yields

$$
\begin{aligned}
& \widehat{F}_{0}(s)=\frac{a}{s+\frac{\lambda_{0}}{r_{0}}-\frac{\mu_{1}}{r_{0}} \widehat{F}_{1}(s)}, \\
& \frac{\hat{F}_{j}(s)}{\widehat{F}_{j-1}(s)}=\frac{\frac{\lambda_{j-1}}{r_{j}}}{s+\frac{\lambda_{j}+\mu_{j}}{r_{j}}-\frac{\mu_{j+1}}{r_{j}} \frac{\hat{F}_{j+1}(s)}{\hat{F}_{j}(s)}}, \quad j=1,2,3, \ldots
\end{aligned}
$$


This leads to the continued fractions

$$
\begin{aligned}
& \hat{F}_{0}(s)=\frac{a}{s+\frac{\lambda_{0}}{r_{0}}-} \frac{\frac{\lambda_{0} \mu_{1}}{r_{0} r_{1}}}{s+\frac{\lambda_{1}+\mu_{1}}{r_{1}}-} \frac{\frac{\lambda_{1} \mu_{2}}{r_{1} r_{2}}}{s+\frac{\lambda_{2}+\mu_{2}}{r_{2}}-} \cdots, \\
& \frac{\hat{F}_{j}(s)}{\hat{F}_{j-1}(s)}=\frac{\frac{\lambda_{j-1}}{r_{j}}}{s+\frac{\lambda_{j}+\mu_{j}}{r_{j}}-} \frac{\frac{\lambda_{j} \mu_{j+1}}{r_{j} r_{j+1}}}{s+\frac{\lambda_{j+1}+\mu_{j+1}}{r_{j+1}}-}-\frac{\frac{\lambda_{j+1} \mu_{j+2}}{r_{j+1} r_{j+2}}}{s+\frac{\lambda_{j+2}+\mu_{j+2}}{r_{j+2}}-} \cdots
\end{aligned}
$$

Define

$$
\phi_{j}(s):=\frac{\frac{\lambda_{j-1}}{r_{j}}}{s+\frac{\lambda_{j}+\mu_{j}}{r_{j}}-} \frac{\frac{\lambda_{j} \mu_{j+1}}{r_{j} r_{j+1}}}{s+\frac{\lambda_{j+1}+\mu_{j+1}}{r_{j+1}}-}-\frac{\frac{\lambda_{j+1} \mu_{j+2}}{r_{j+1} r_{j+2}}}{s+\frac{\lambda_{j+2}+\mu_{j+2}}{r_{j+2}}-} \cdots
$$

Then,

$$
\hat{F}_{0}(s)=\frac{a}{s+\frac{\lambda_{0}}{r_{0}}-\frac{\mu_{1}}{r_{0}} \phi_{1}(s)}, \quad \frac{\hat{F}_{j}(s)}{\widehat{F}_{j-1}(s)}=\phi_{j}(s) .
$$

After certain algebra, we obtain

$$
\begin{aligned}
& \hat{F}_{0}(s)=a \sum_{k=0}^{\infty}\left(\frac{\mu_{1}}{r_{0}}\right)^{k} \frac{\left(\phi_{1}(s)\right)^{k}}{\left(s+\frac{\lambda_{0}}{r_{0}}\right)^{k+1}}, \\
& \hat{F}_{j}(s)=\left(\prod_{k=1}^{j} \phi_{k}(s)\right) \hat{F}_{0}(s), \quad j=1,2,3, \ldots
\end{aligned}
$$

On inversion, we get

$$
\begin{aligned}
& F_{0}(u)=a \sum_{k=0}^{\infty}\left(\frac{\mu_{1}}{r_{0}}\right)^{k}\left(\frac{u^{k} e^{-\left(\lambda_{0} / r_{0}\right) u}}{k !}\right) * \phi_{1}^{*(k)}(u), \\
& F_{j}(u)=\phi_{1}(u) * \phi_{2}(u) * \cdots * \phi_{j}(u) * F_{0}(u), \quad j=1,2,3, \ldots
\end{aligned}
$$

In the following argument we consider a specific nature of birth and death rates. 


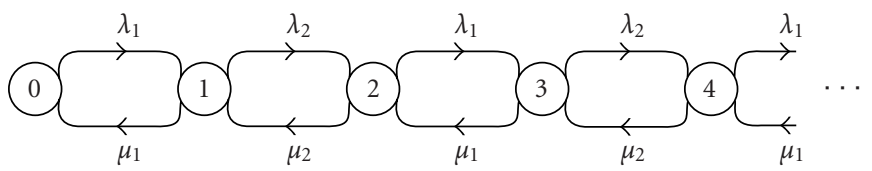

Figure 4.1. State-transition diagram of the background Markov process.

Example 4.1. Consider a fluid queue driven by an $M / M / 1$ queue with two alternating arrival rates and two alternating service rates as shown in Figure 4.1.

We assume the net input rate of fluid to be $r_{0}<0, r_{2 j}=r_{1}>0$ for $j=1,2,3, \ldots$ and $r_{2 j+1}=r_{2}>0$ for $j=0,1,2, \ldots$. The steady state probabilities are given by (3.25) with $p_{0}=\left(\mu_{1} \mu_{2}-\lambda_{1} \lambda_{2}\right) / \mu_{2}\left(\lambda_{1}+\mu_{1}\right)$. From (2.3), the condition $\mu_{1}\left(r_{0}+r_{1}\right)+\lambda_{1} r_{2}<0$ ensures the stability of the process $\{(X(t), C(t)), t \geq 0\}$. For the model under consideration,

$$
\phi_{j}(s)= \begin{cases}f(s), & \text { if } j \text { is odd } \\ g(s), & \text { if } j \text { is even, }\end{cases}
$$

where

$$
\begin{gathered}
f(s)=\frac{\frac{\lambda_{1}}{r_{2}}}{s+\frac{\lambda_{2}+\mu_{1}}{r_{2}}-} \frac{\frac{\lambda_{2} \mu_{2}}{r_{1} r_{2}}}{s+\frac{\lambda_{1}+\mu_{2}}{r_{1}}-} \frac{\frac{\lambda_{1} \mu_{1}}{r_{1} r_{2}}}{s+\frac{\lambda_{2}+\mu_{1}}{r_{2}}-} \\
=\frac{\lambda_{1}}{r_{2} s+\lambda_{2}+\mu_{1}-\frac{\lambda_{2} \mu_{2}}{r_{1} s+\lambda_{1}+\mu_{2}-\mu_{1} f(s)}}
\end{gathered}
$$

Or

$$
\begin{aligned}
& \mu_{1}\left(r_{2} s+\lambda_{2}+\mu_{1}\right)(f(s))^{2} \\
& \quad-\left[\left(r_{1} s+\lambda_{1}+\mu_{2}\right)\left(r_{2} s+\lambda_{2}+\mu_{1}\right)-\lambda_{2} \mu_{2}+\lambda_{1} \mu_{1}\right] f(s)+\lambda_{1}\left(r_{1} s+\lambda_{1}+\mu_{2}\right)=0 .
\end{aligned}
$$

Using Rouches theorem, considering the root that lies inside the unit circle, we get

$$
\begin{gathered}
f(s)=\frac{1}{2 \mu_{1}\left(r_{2} s+\lambda_{2}+\mu_{1}\right)}\left[\left(r_{1} s+\lambda_{1}+\mu_{2}\right)\left(r_{2} s+\lambda_{2}+\mu_{1}\right)+\lambda_{1} \mu_{1}-\lambda_{2} \mu_{2}\right. \\
+\left(\left[\left(r_{1} s+\lambda_{1}+\mu_{2}\right)\left(r_{2} s+\lambda_{2}+\mu_{1}\right)+\lambda_{2} \mu_{2}-\lambda_{1} \mu_{1}\right]^{2}\right. \\
\left.\left.\quad-4 \lambda_{1} \mu_{1}\left(r_{1} s+\lambda_{1}+\mu_{2}\right)\left(r_{2} s+\lambda_{2}+\mu_{1}\right)\right)^{1 / 2}\right] .
\end{gathered}
$$


$g(s)$ is obtained from $f(s)$ by interchanging $r_{1}$ and $r_{2}, \lambda_{1}$ and $\lambda_{2}$, and $\mu_{1}$ and $\mu_{2}$. The expression for $f(s)$ can be written as

$$
f(s)=\frac{r_{1} r_{2}\left[(s+a)^{2}-b^{2}\right]-\sqrt{r_{1}^{2} r_{2}^{2}\left((s+a)^{2}-b^{2}\right)^{2}-4 \lambda_{1} \mu_{1} r_{1} r_{2}\left((s+a)^{2}-c^{2}\right)}}{2 \mu_{1} r_{2}((s+a)-c)},
$$

where

$$
\begin{aligned}
a & =\frac{1}{2}\left(\frac{\lambda_{1}+\mu_{2}}{r_{1}}+\frac{\lambda_{2}+\mu_{1}}{r_{2}}\right), \\
b^{2} & =a^{2}-\left(\frac{\lambda_{1} \lambda_{2}+\mu_{1} \mu_{2}+2 \lambda_{1} \mu_{1}}{r_{1} r_{2}}\right), \\
c & =\frac{1}{2}\left(\frac{\lambda_{1}+\mu_{2}}{r_{1}}-\frac{\lambda_{2}+\mu_{1}}{r_{2}}\right) .
\end{aligned}
$$

On inversion, we get

$$
f(u)=2\left(\frac{\lambda_{1}}{r_{2}}\right) e^{-a u}\left\{f_{2}(u) * \sum_{n=0}^{\infty}(-1)^{n} \sum_{k=0}^{n} \frac{(n / 2)(n / 2-1) \cdots(n / 2-k+1)}{k !} f_{3}^{*(k)}(u)\right\},
$$

where

$$
\begin{aligned}
& f_{3}(u)=\left(\frac{4 \lambda_{1} \mu_{1}}{r_{1} r_{2}}\right)\left(f_{1}(u) * f_{2}(u)\right), \\
& f_{1}(u)=\cosh b u+\frac{c}{b} \sinh b u, \\
& f_{2}(u)=\cosh b u-\frac{c}{b} \sinh b u,
\end{aligned}
$$

and $h^{*(k)}(u)$ is the $k$-fold convolution of $h(u)$ with itself. Also, from (4.5),

$$
\frac{\widehat{F}_{2 k+1}(s)}{\hat{F}_{2 k}(s)}=f(s), \quad \frac{\widehat{F}_{2 k}(s)}{\widehat{F}_{2 k-1}(s)}=g(s),
$$

which on solving yield

$$
\begin{aligned}
\widehat{F}_{2 k+1}(s) & =(f(s))^{k+1}(g(s))^{k} \widehat{F}_{0}(s), \\
\hat{F}_{2 k}(s) & =(f(s))^{k}(g(s))^{k} \widehat{F}_{0}(s) .
\end{aligned}
$$




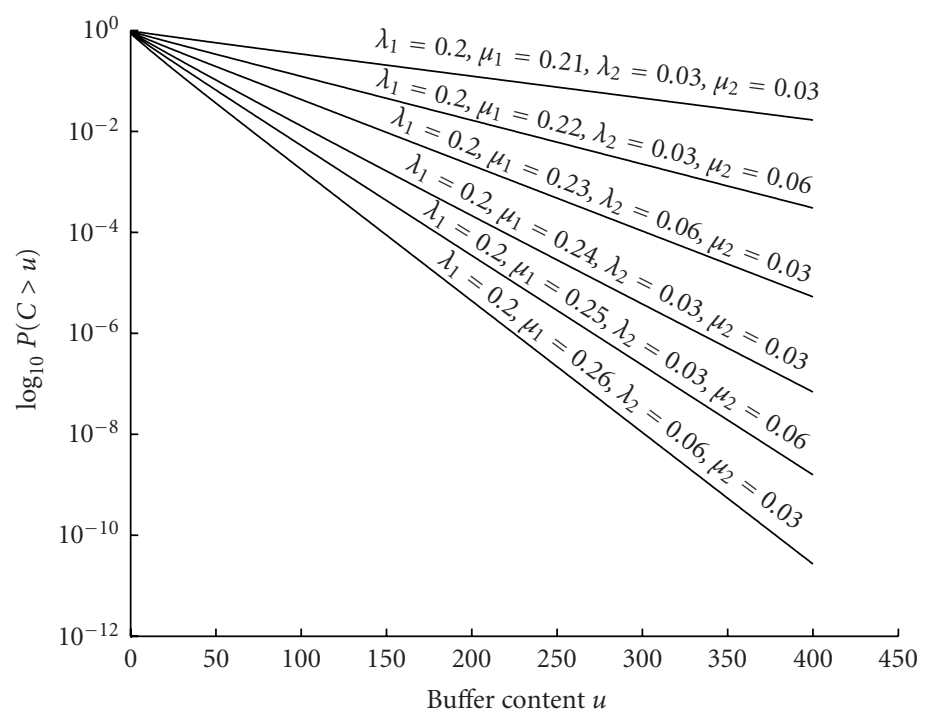

Figure 5.1. Buffer content distribution on a finite state space with $N=21, r_{1}=-1$, and $r_{2}=1$.

On inversion

$$
\begin{aligned}
F_{2 k+1}(u) & =f^{*(k+1)}(u) * g^{*(k)}(u) * F_{0}(u), \quad k=0,1,2, \ldots, \\
F_{2 k}(u) & =f^{*(k)}(u) * g^{*(k)}(u) * F_{0}(u), \quad k=1,2,3, \ldots,
\end{aligned}
$$

where $F_{0}(u)$ is obtained from $(4.7)$ as

$$
F_{0}(u)=a \sum_{k=0}^{\infty}\left(\frac{\mu_{1}}{r_{0}}\right)^{k}\left(\frac{u^{k} e^{-\left(\lambda_{0} / r_{0}\right) u}}{k !}\right) * f^{*(k)}(u)
$$

\section{Numerical illustrations}

In this section, we provide numerical examples for the fluid queue driven by the finite and infinite queuing model discussed in the earlier sections.

In Figure 5.1 the buffer overflow probability corresponding to the varying values of the parameters $\lambda_{1}, \lambda_{2}, \mu_{1}$, and $\mu_{2}$ is plotted against buffer size for finite state space with $N=21, r_{1}=-1$, and $r_{2}=1$ (see Remark 3.4). The overflow probabilities are found to decrease rapidly with increase in $\mu_{1}$. The graphs are plotted for two sets of parameters by considering the three different cases wherein the ratio $\lambda_{2} / \mu_{2}$ is equal to one, less than one, and greater then one, respectively.

Figure 5.2 depicts an analogous setting on an infinite state space with $N$ truncated at $25, r_{0}=-1$, and $r_{i}=1$ for $i \geq 1$. For constant values of $\lambda_{1}$ and $\lambda_{2}$, the overflow probabilities decrease rapidly with increase in $\mu_{1}$. The overflow probability is in the range of $10^{-10}$ to $10^{-15}$ and hence becomes a rare event. 


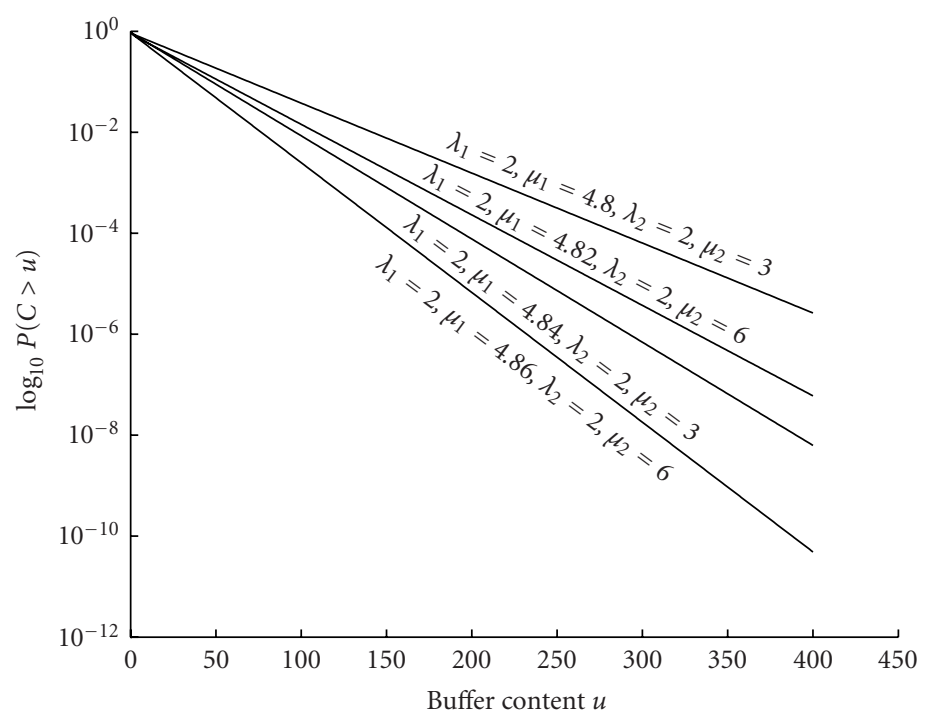

Figure 5.2. Buffer content distribution on an infinite state space with $N$ truncated at $25, r_{0}=-1$, and $r_{1}=1=r_{2}$.

\section{Appendix}

\section{Some identities of tridiagonal determinants}

Here we give some identities of tridiagonal determinants (Muir [4]), which are useful in determining the stationary buffer content distribution of the model under consideration.

Identity A.1.

$$
\begin{aligned}
& \left|\begin{array}{ccccc}
A+a_{0} & a_{0} & & & \\
b_{1} & A+a_{1}+b_{1} & & & \\
& & \ddots & & \\
& & & A+a_{n-1}+b_{n-1} & a_{n-1} \\
& & & b_{n} & A+b_{n}
\end{array}\right|_{n+1} \\
& =A \times\left|\begin{array}{cccccc}
g_{1} & b_{1} & & & & \\
a_{1} & g_{2} & b_{2} & & & \\
& & & \ddots & & \\
& & & a_{n-2} & g_{n-1} & b_{n-1} \\
& & & & a_{n-1} & g_{n}
\end{array}\right|_{n},
\end{aligned}
$$

where $g_{j}=A+a_{j-1}+b_{j}, j=1,2, \ldots, n$. 
Identity A.2.

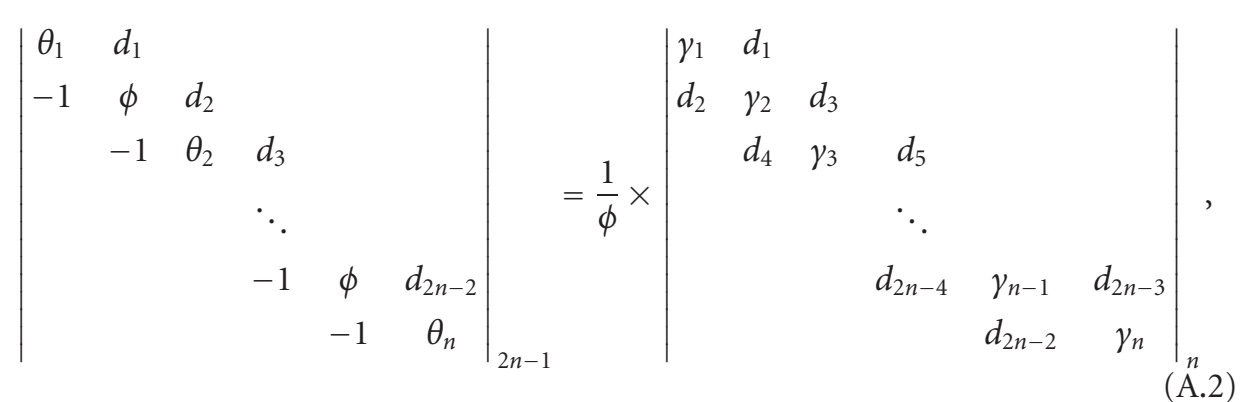

where $\gamma_{j}=d_{2 j-2}+\theta_{j} \phi+d_{2 j-1}, j=1,2, \ldots, n$, with $d_{0}=0$ and $d_{2 n-1}=0$.

Identity A.3.

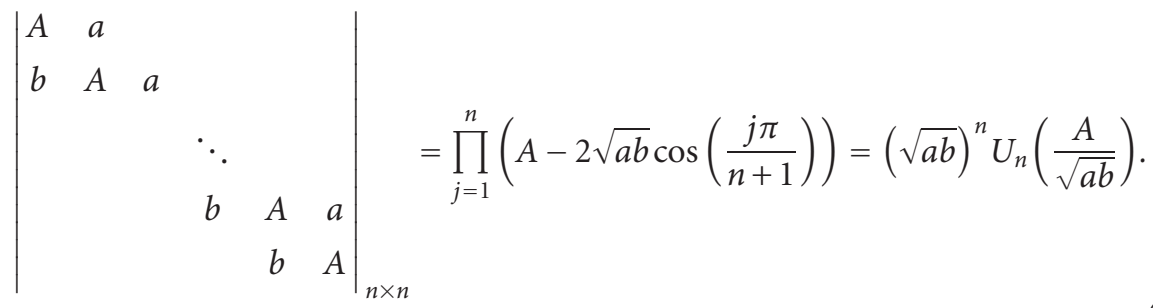

Identity A.4.

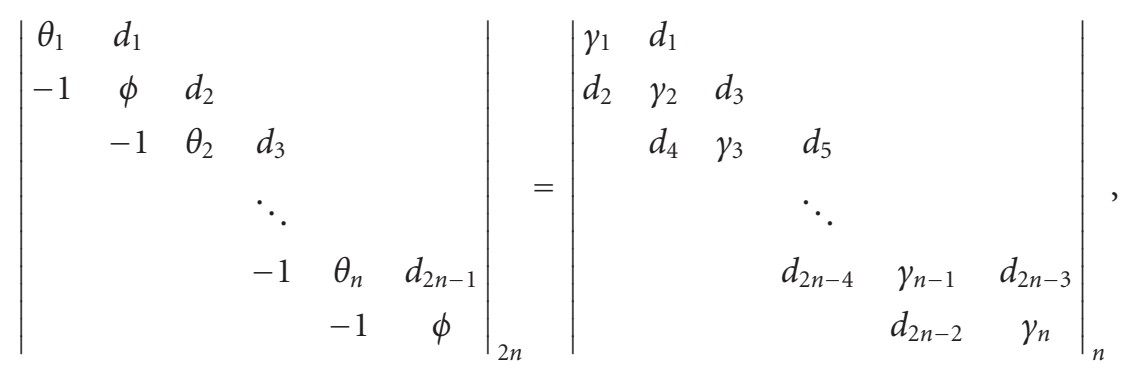

where $\gamma_{j}=d_{2 j-2}+\theta_{j} \phi+d_{2 j-1}, j=1,2, \ldots, n$, with $d_{0}=0$.

\section{References}

[1] G. Horton, V. G. Kulkarni, D. M. Nicol, and K. S. Trivedi, Fluid stochastic Petri nets: theory, applications, and solution techniques, European J. Oper. Res. 105 (1998), no. 1, 184-201.

[2] R. B. Lenin and P. R. Parthasarathy, Fluid queues driven by an $M / M / 1 / N$ queue, Math. Probl. Eng. 6 (2000), no. 5, 439-460.

[3] D. Mitra, Stochastic theory of a fluid model of producers and consumers coupled by a buffer, Adv. Appl. Probab. 20 (1988), no. 3, 646-676.

[4] T. Muir, A Treatise on the Theory of Determinants, Dover Publications, New York, 1960, revised and enlarged by William H. Metzler. 
[5] E. A. van Doorn and W. R. W. Scheinhardt, A fluid queue driven by an infinite-state birth-death process, Proc. 15th International Teletraffic Congress (V. Ramaswami and P. E. Wirth, eds.), Elsevier, Amsterdam, 1997, pp. 465-475.

[6] W. Whitt, Stochastic-Process Limits. An Introduction to Stochastic-Process Limits and Their Application to Queues, Springer Series in Operations Research, Springer-Verlag, New York, 2002.

P. R. Parthasarathy: Department of Mathematics, Indian Institute of Technology, Madras, Chennai 600 036, India

E-mail address: prp@iitm.ac.in

K. V. Vijayashree: Department of Mathematics, Indian Institute of Technology, Madras, Chennai 600036 , India

E-mail address: ma99p01@violet.iitm.ernet.in

R. B. Lenin: Department of Mathematics and Computer Science, University of Antwerp, Universiteitsplein 1, 2610 Antwerpen, Belgium

E-mail address: lenin@uia.ua.ac.be 


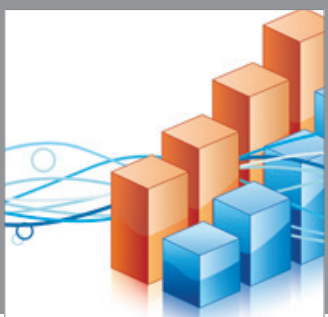

Advances in

Operations Research

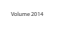

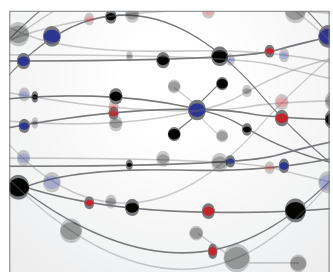

\section{The Scientific} World Journal
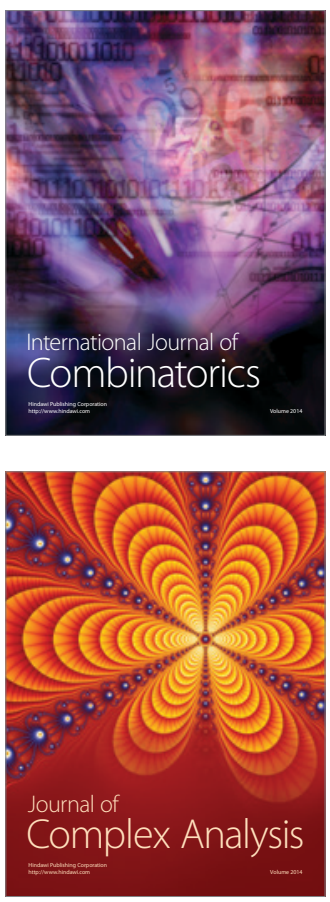

International Journal of

Mathematics and

Mathematical

Sciences
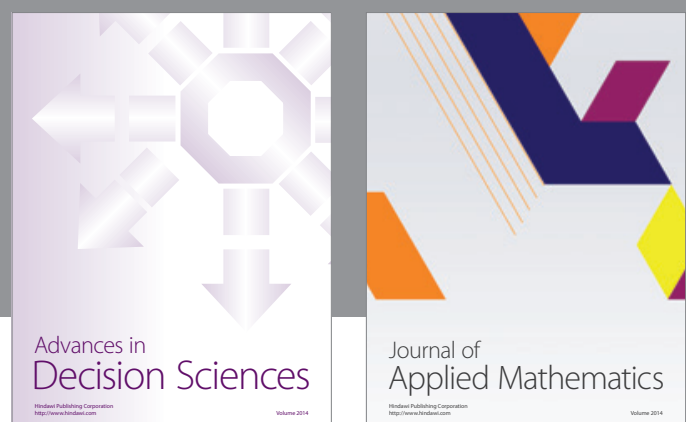

Journal of

Applied Mathematics
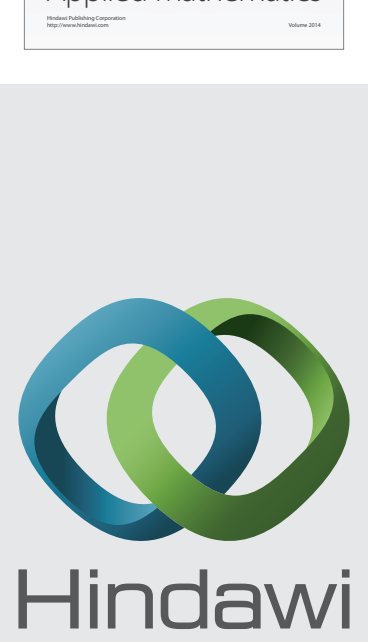

Submit your manuscripts at http://www.hindawi.com
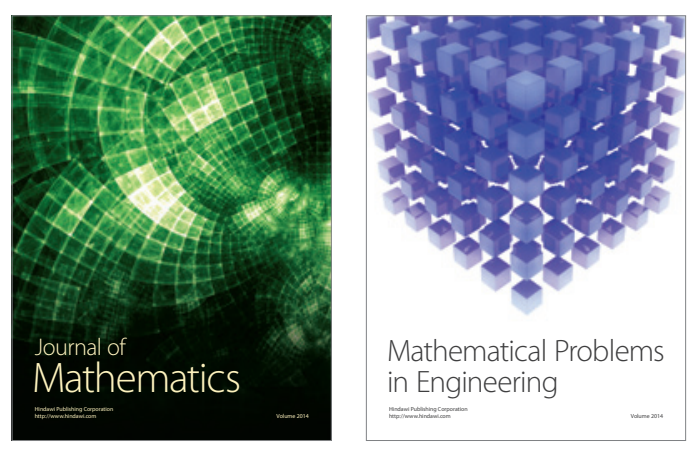

Mathematical Problems in Engineering
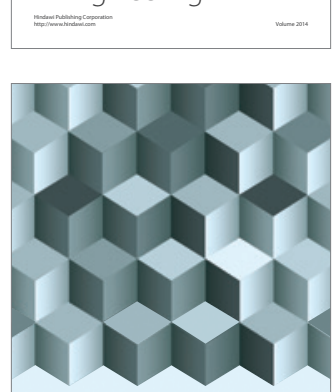

Journal of

Function Spaces
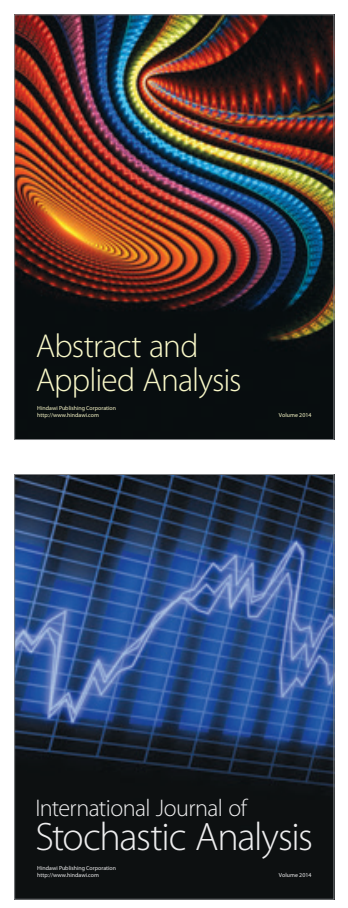

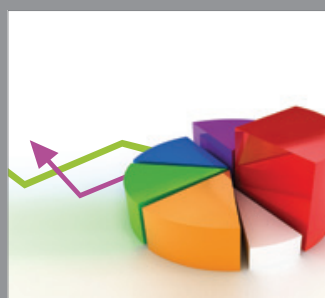

ournal of

Probability and Statistics

Promensencen
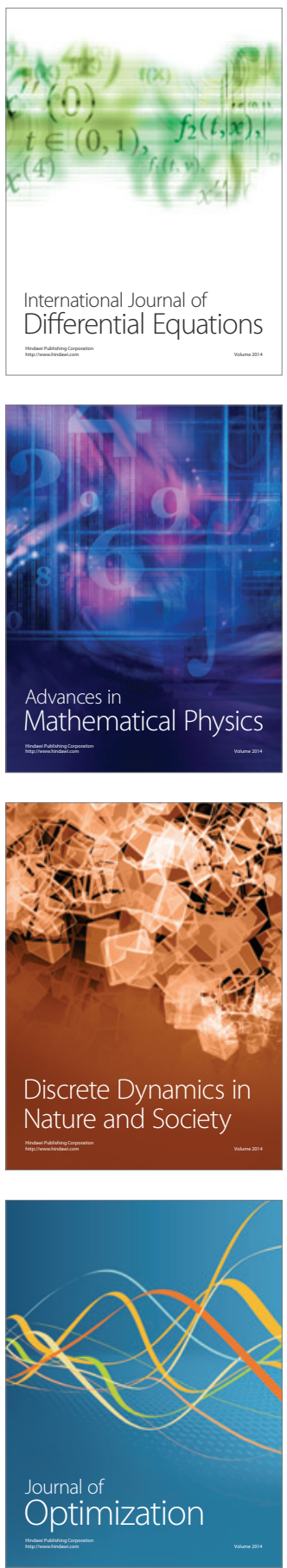